\title{
DINÁMICA SOCIOECONÓMICA, USO DE SUELO MULTITEMPORAL-ESPACIAL Y SU IMPACTO AMBIENTAL EN EL RECURSO HÍDRICO DE LA MICROCUENCA DEL RÍO BILAMPÍ, MUNICIPIO DE RÍO BLANCO, DEPARTAMENTO MATAGALPA, NICARAGUA, I SEMESTRE 2013
}

Ingrid Elizabeth Úbeda Trujillo y Lisseth Blandón Chavarría*

\section{Resumen}

La investigación se realizó en el municipio de Río Blanco en las coordenadas UTM 1438714 Norte 0690735 Oeste, para evaluar la dinámica socioeconómica se aplicó un censo a las 70 familias distribuidas en la microcuenca, todas ellas dedicadas a la agricultura extensiva con bajos niveles tecnológicos que actualmente influyen en el deterioro del recurso suelo y agua.

En la base cartográfica de uso de suelo elaborada por INETER (año 2006) se georeferenciaron las áreas productivas (fincas) ubicadas en la parte alta, media y baja del área en estudio obteniéndose el mapa de uso de suelo actual del año 2013, posteriormente en el análisis multitemporal de coberturas de uso de suelo (bosque latofoliado y tierras agropecuarias) de acuerdo a los años 1983, 1990,2001,2006, se detectó una tasa de cambio de 450 ha de bosque latifoliado que fueron sustituidas por tierras agropecuarias.

Del mapa de uso actual y uso potencial se identificó que el área presenta un índice de tierras sobreutilizada del $40 \%$, uso adecuado de $39 \%$ y subutilizado de $21 \%$, el primer dato indica que está siendo explotada por encima del potencial establecido (forestal).

Para valorar los impactos ambientales en el recurso hídrico se analizó las implicaciones ambientales y se aplicó un instrumento más que consiste en la toma de una muestra de agua en la parta baja de la microcuenca la misma se trasladó CIRA-UNAN para ser sometidas a análisis de tipo plaguicidas organoclorado y organofosforado.

Palabras claves: Uso de suelo multitemporal-espacial, Georeferenciacion, microcuenca, impacto ambiental, Recursos Hídricos, socioeconómico.

\section{Abstract}

The research was conduted in the municipality of Rio Blanco, Matagalpa at coordinates UTM north 1437714 y 690735 west, to assess the socicioeconomic diynamics census was applied to 70 families distrubuted in the wastershed, all dedicated to extensive agriculture and low levels of technoly that currently influence in the deterioration of soil and water resources.

On the basis of land mapping produced by INETER (year 2006) were georeferenced productive areas (farm) located in the upper, middle and lower yielding the study área map of current land use in 2013, then in the multitemporal analysis of land use covarage (latifoliado forest and agricultural land)

Lic. Ingrid Elizabeth Úbeda Trujillo,

Lic. Lisseth Blandón Chavarría.

Docentes instructores de cátedra del departamento de Geografía, UNAN-Managua. 
according to the years 1983, 1990, 2001, 2006, we detected and Exchange rate of 450 ha of broadleaf forwst were replaced by agricultural land.

Map of current use and potential use was indentified tha the area has and index of $40 \%$ overused land proper use underutilized $39 \%$ and $21 \%$, indicates that the first data is being exploited above potential established (forest)

To assess the environmental impacts on water resources environmental implications analyzed and applied another instrument that involves taking a simple of water in the bottom of the watershed, it was transmitted CIRA-UNAN to be subjeted to analysis organochlorine and orgaphosphorus pesticide type.

Keyword: Land use multitemporal-spacial, georeferencing, watershed, environmental, water resources, socioeconomic, Universal Transversal of Mercartor (UTM).

\section{Introducción}

L a microcuenca Bilampí pertenece a la cuenca del Río Grande de Matagalpa (cuenca número 55), considerada el segundo cauce fluvial más extenso, ubicado en el centro de Nicaragua drenando sus aguas al mar Caribe.

La investigación que presentamos es pertinente debido a que la microcuenca del río Bilampí forma parte del área protegida cerro Musún y según la Fundación Nicaragüense para el Desarrollo Sostenible (FUNDENIC) cumple con las siguientes funciones: hidrológica, ambiental $y$ socioeconómica.

Se han realizado estudios de carácter geológico, ambiental e hídrico por parte de instituciones como: INETER, FUNDENIC, COSUDE Y NITLAPLAN-

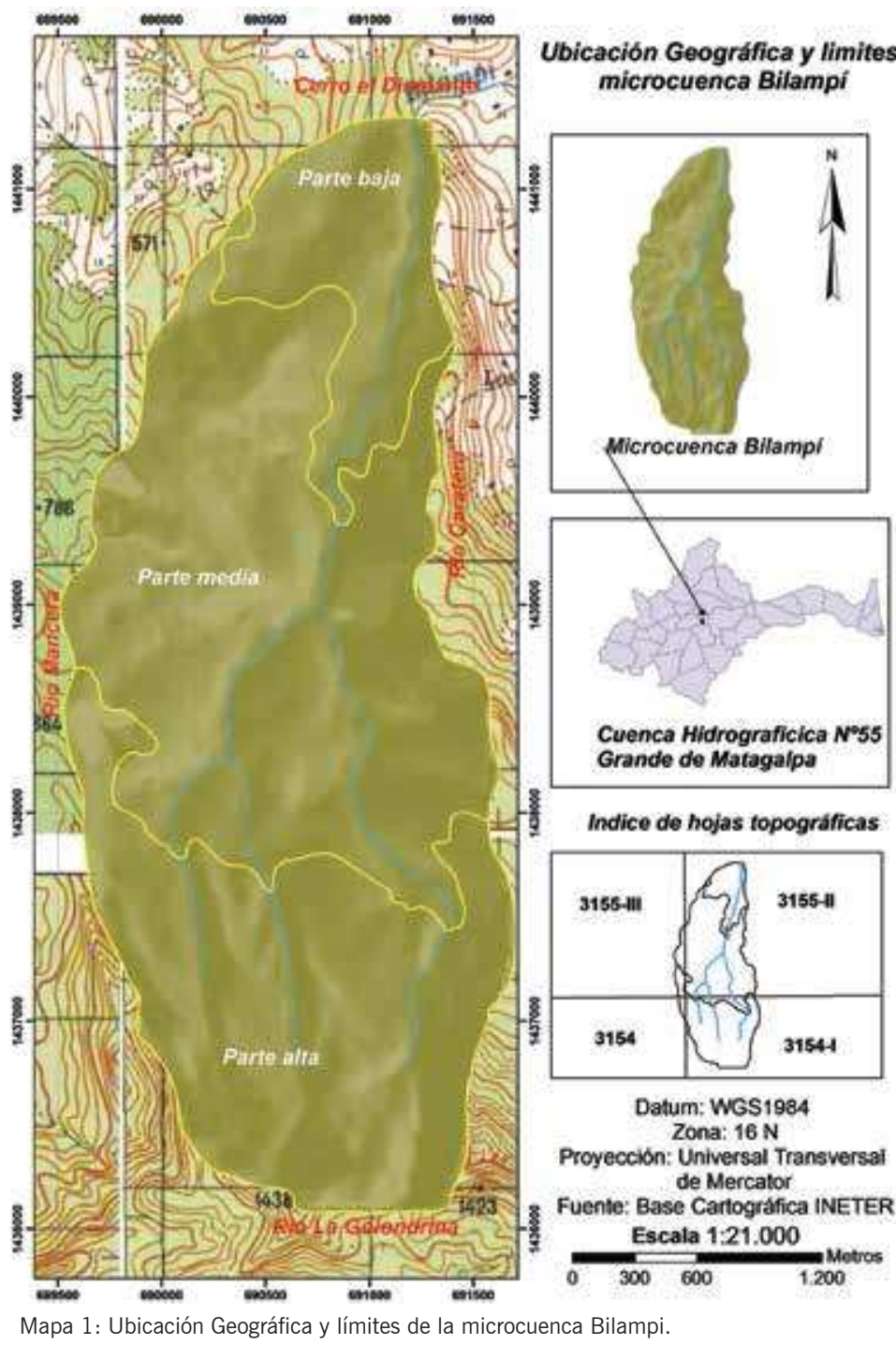


UCA y UNAN-Managua. Sin embargo, en el aspecto socioeconómico, usos de suelo multitemporal-espacial y los impactos ambientales en el recurso hídrico, son pocas las consideraciones conocidas relacionadas a estos temas.

Estas consideraciones se justifican plenamente ya que los resultados del estudio facilitarían la planeación y gestión integrada de recursos hídricos, que emprendan los diferentes actores e instituciones interesadas en la temática.

\section{Ubicación geográfica de la microcuenca}

La microcuenca Bilampí forma parte de la cuenca grande de Matagalpa ( ${ }^{\circ} 55$ ), específicamente se ubica en la subcuenca Bilampí del municipio de Río Blanco, departamento de Matagalpa. Con una superficie de $8.1 \mathrm{~km}(810 \mathrm{ha})$ y un perímetro de $13 \mathrm{~km}$ se localiza en las coordenadas UTM 1438714 Norte y 690735 Oeste.

\section{Límites de la microcuenca:}

Norte: Cerro el Diamante

Sur: Río La Golondrina.

Este: Río Caratera.

Oeste: Río Mancera.

Características morfométricas de la microcuenca Bilampí

\begin{tabular}{|c|c|c|}
\hline Datos & Dimensión & Descripción \\
\hline \multicolumn{2}{|l|}{ Parámetros de forma } & \\
\hline Coeficiente de compacidad & 1.3 & oval redonda a oval oblonga \\
\hline \multicolumn{2}{|l|}{ Parámetros de relieve } & \\
\hline Área & 810 ha & Muy pequeña \\
\hline Perímetro & $13 \mathrm{~km}$ & \\
\hline Altitud media de la microcuenca & 878 m.s.n.m & \\
\hline \multicolumn{2}{|l|}{ Parámetros de red de drenaje } & \\
\hline Densidad de drenaje & $1.2 \mathrm{Km}^{2}$ & Baja \\
\hline Altitud máxima & 1420 m.s.n.m & \\
\hline Altitud mínima & 280 m.s.n.m & \\
\hline Longitud del cauce principal & $3 \mathrm{Km}$ & \\
\hline Pendiente media del río Bilampí & 460 m.s.n.m & \\
\hline Orden de la corriente & 1,2 orden & Perenne e efímeras \\
\hline Frecuencia de causes & $0.375 \mathrm{~km}$ & \\
\hline
\end{tabular}

Tabla 1, Fuente: Propia 


\section{Hidrografía}

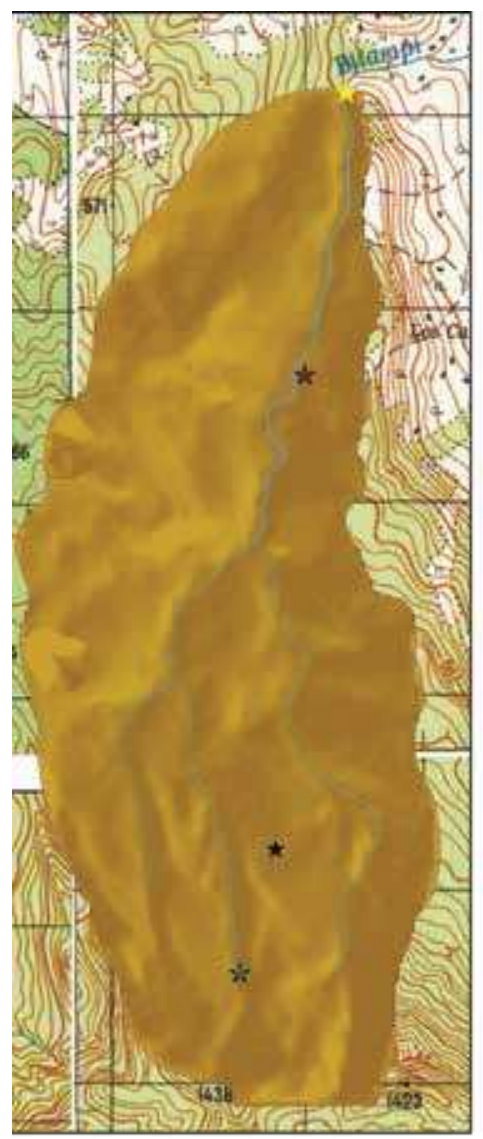

Mapa 2: Características Morfométrica.
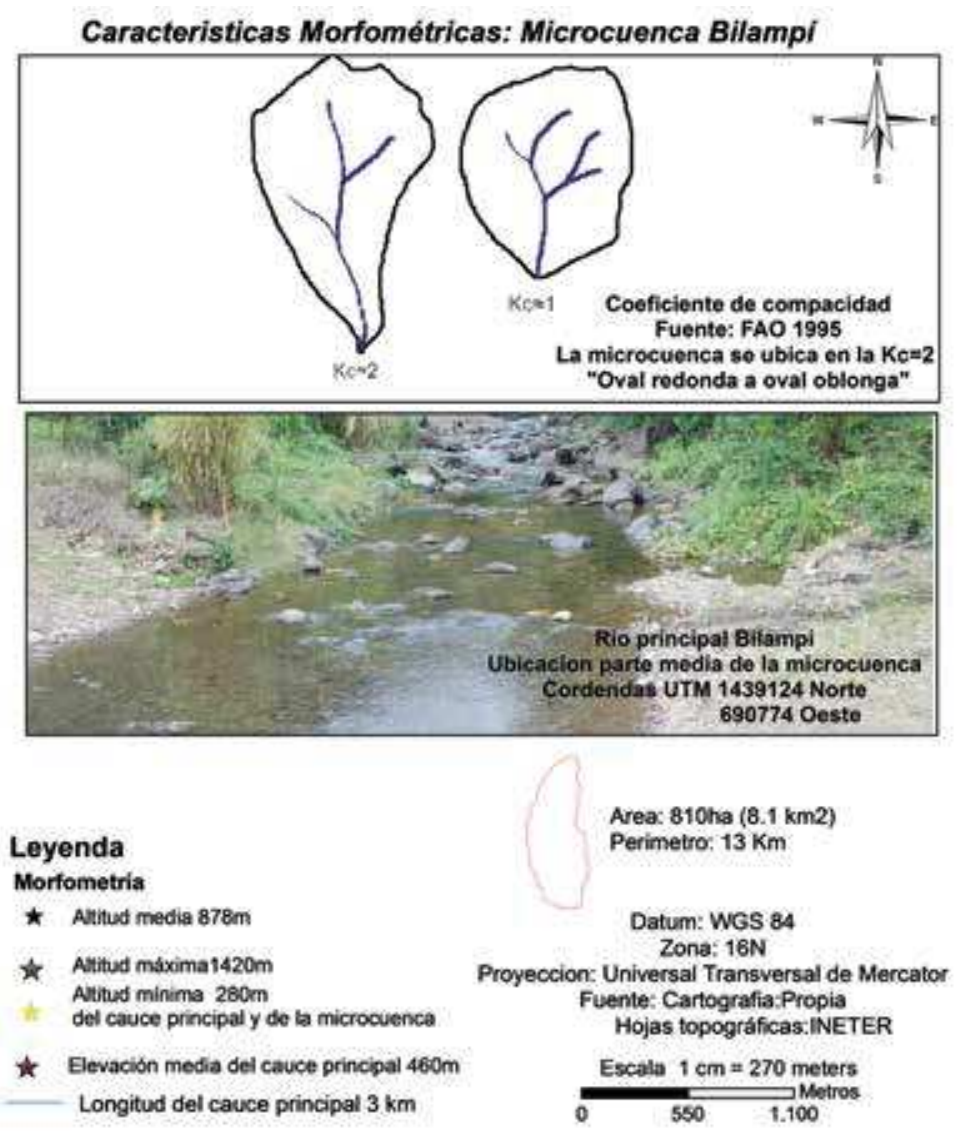

La microcuenca Bilampí, ocupa un área de aproximadamente $8.1 \mathrm{Km}^{2}$ (801 ha), por consiguiente la red hidrográfica esta poco jerarquizada y la ramificación de los tributarios (cauces) es de tipo dendrítica ${ }^{1}$, esto significa que estos tributarios nacen en la cota de elevación de los 1200 m, coincidiendo con la parte alta de la microcuenca, donde el orden de las corrientes son de tipo efímeras ${ }^{2}$ (nacimiento del río Bilampí) y la cobertura vegetal es de tipo tropical latifoliado montano bajo.
De acuerdo a las características morfométricas, la microcuenca Bilampí se divide en parte alta, media y baja e hidrográficamente la red de drenaje está integrada por el río principal Bilampí y tres tributarios (efímeros). Estos últimos presentan un sistema de drenaje de primer orden, ubicándose en la parte alta de la microcuenca y alimentando al río principal Bilampí que es de segundo orden. La longitud del río principal Bilampí es de $3 \mathrm{Km}$, la longitud total de

Criterío establecido por Horton 1945

2 Criterío establecido por Horton 1945 


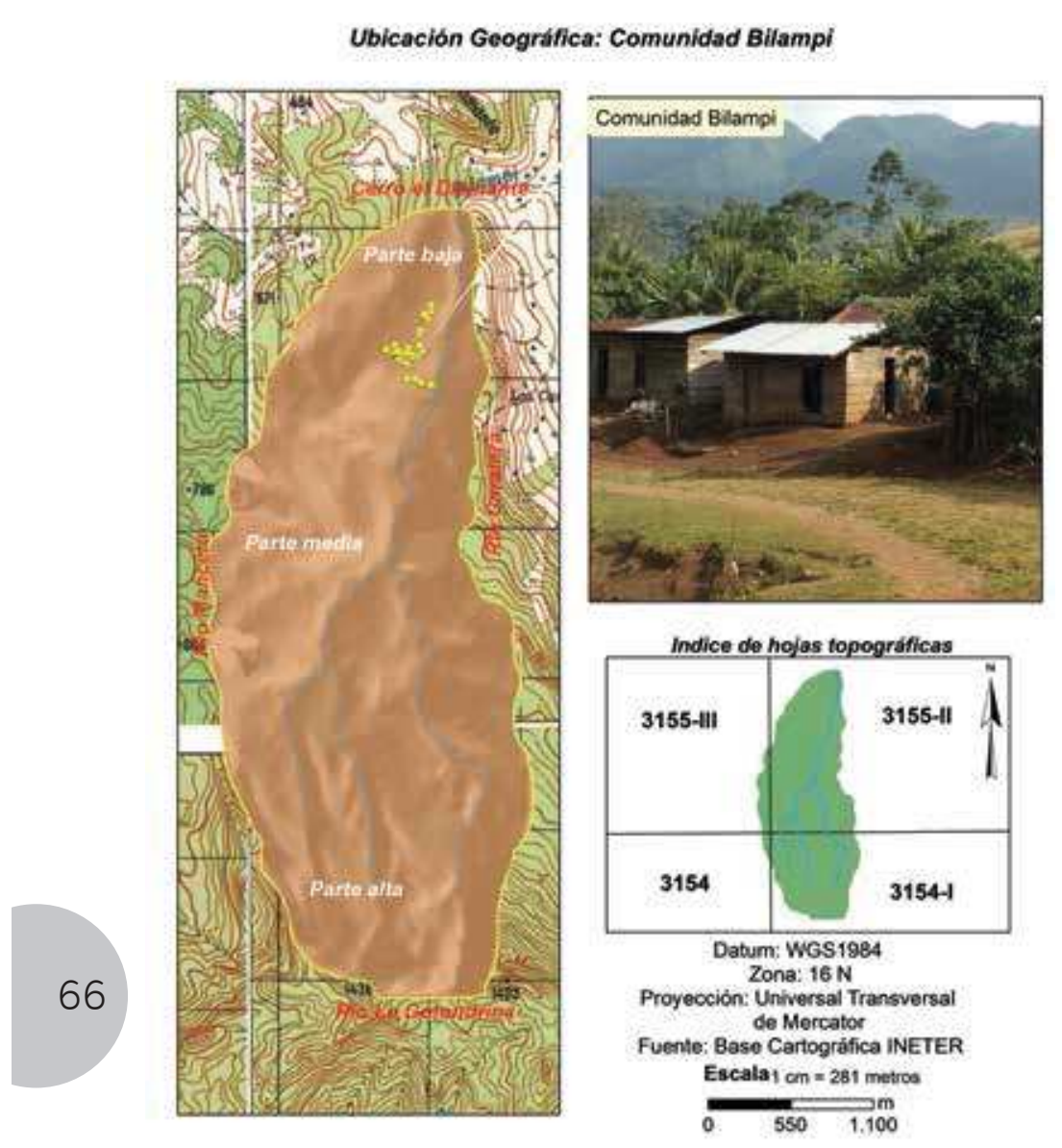

educativo de educación primaria que atiende la modalidad de 1er a 6to grado.

De acuerdo a las características sanitarias la comunidad no cuenta con alcantarillado sanitario $\mathrm{y}$ letrinas. Los comités de agua potable y saneamiento (CAPS) se han ubicado en algunas comunidades; entre ellas, Bilampí permitiendo que el $58.58 \%$ (41 viviendas) tengan acceso a agua que es suministrados a través de un puesto público. El 41.4\% (29 viviendas) se abastecen de manantiales cercanos sus viviendas.

El servicio de energía eléctrica es suministrado a través de la producción de agua que genera este río y sus afluentes, existe una vía principal de comunicación es una carretera de todo tiempo no pavimentada (Río Blanco-Bilampí).

\section{Actividades económicas}

En el gráfico se observa, que existe un predominio de la actividad primaria, representado por el 98\%, distribuidos en 68 agricultores, 19 ganaderos, y 2 jornaleros. Las actividades económicas en la comunidad se han visto regidas primordialmente por este sector económico, siendo la principal fuente de ingresos de las unidades familiares rurales. La actividad terciaria es mínima ( $2 \%)$, es representada por el comercio de granos básicos que abastece el mercado local, departamental y autoconsumo familiar.

$\%)$ y 152 mujeres (44\%). Existe un centro 


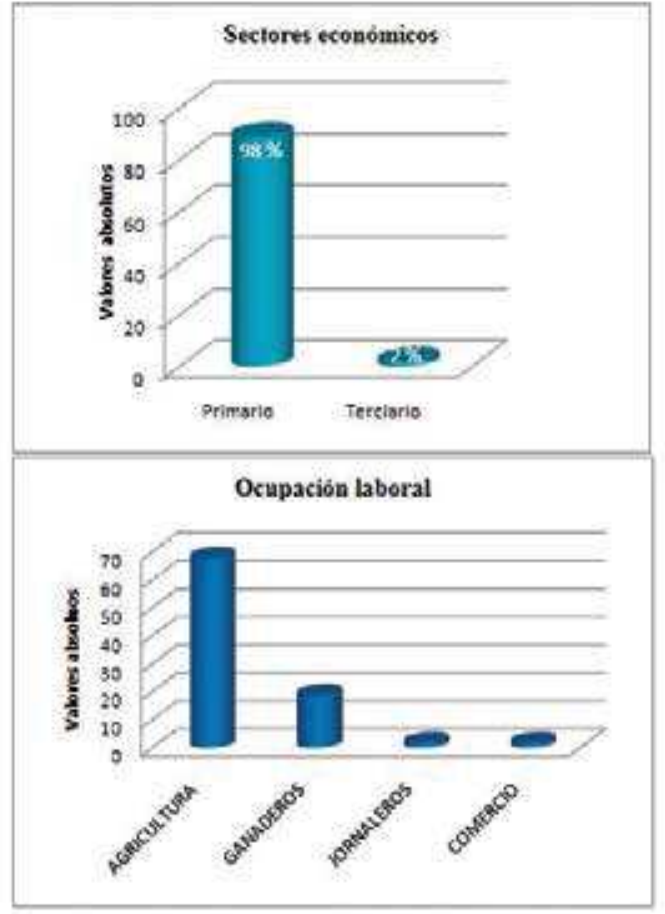

Gráfico 1: Sectores económicos

- FRIIOLESY MAAZ WCACAO, MUSACEASY TUBERCULOS

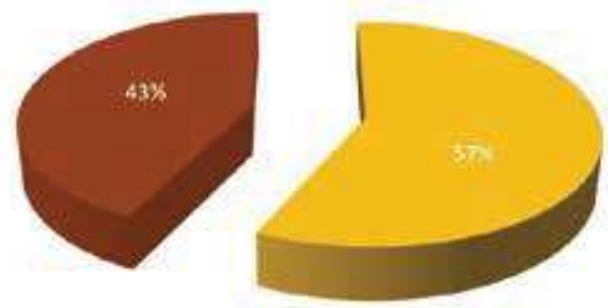

Gráfico 2: Porcentaje de cultivos

De los tipos de cultivos existentes en la microcuenca en estudio, el maíz y el frijol representan el $57 \%$ de la producción $\mathrm{y}$ en menor escala el cacao, musácea y tubérculos (quequisque, malanga, yuca o mandioca) representan el $43 \%$.

Del total de agroquímicos detallados en la tabla 7 , los cinco primeros pertenece a la llamada docena sucia (docena maldita), responsable de daños en los animales, medio ambiente e imperfecciones en la salud.
De acuerdo a entrevistas realizadas, algunos productores utilizan plaguicidas como el malation Clorpirifo, Diazinon y Endulsufan para combatir las moscas y los insectos en cultivos maíz y frijoles.

\section{Cambios de uso de suelo en la microcuenca Bilampí en el período de los años 1983-2013}

Basado en el mapa de uso potencial del municipio de Río Blanco elaborado por SINAPRED, INETER y COSUDE, se clasificó el uso potencial en la micro cuenca Bilampí, de acuerdo a características, propiedades de suelo, relieve y condiciones ambientales, siendo de tipo forestal (F). De acuerdo a las características de profundidad y textura fina, estos suelos son aptos para sistemas de producción agroforestal con pendientes menores del $50 \%$, en pendientes de $0-30 \%$ para silvopastoríl y de $0-50 \%$ para forestal, por tanto en la microcuenca Bilampí, los suelos son aptos para la producción forestal.

\section{A continuación se muestra un mapa con detección de cambios de uso de suelo de los años 1983-2013}

Se observa que en la clase Bosque latifoliado $(\mathrm{Bl})$ ha disminuido su representación espacial, teniendo en 1983 780 ha, ocupando la parte alta, media y baja de la microcuenca y actualmente cubre 330ha localizados solamente en la parte alta, lo cual significa la pérdida 450ha. En cambio la clase Ta tiende a aumentar de 30 ha a 480ha, ambas clases en un período de 30 años.

En el año 1983 la cobertura vegetal de Bosque latifoliado (Bl) cubría 96\% del área total de la microcuenca, esto se debe a la baja densidad poblacional en la zona. En entrevista a la Sra. Antonia Díaz Martínez, 


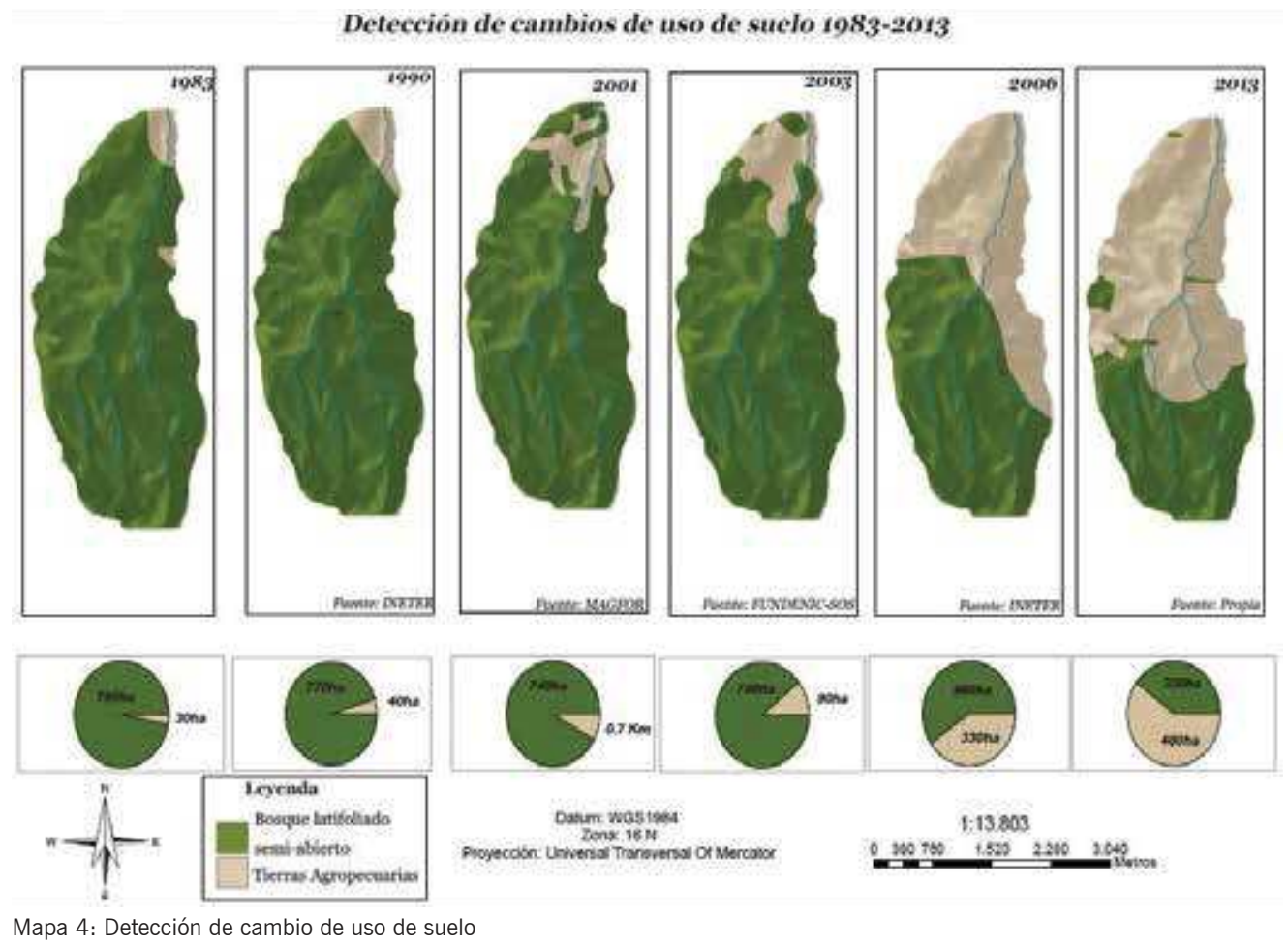

comentaba que los primeros pobladores en el año de 1972 lo conformaban tres familias, aumentando a 11 para el año 1983, asentados estos comunitarios en la parte baja.

Hasta el 2003 la clase Bl no había disminuido significativamente y la clase Tierra agropecuaria (Ta) era una actividad económica incipiente, debido a que no había áreas productivas (Fincas) de gran extensión y la mayoría de los propietarios cultivaban para satisfacer las necesidades familiares. El aumento repentino de la población en año 2004 (deslizamiento), provocó desplazamientos de personas a la parte baja de la microcuenca Bilampíi ${ }^{3}$, por ello, en un período de seis años (2006-2013) hubo un aumento en la forma tenencia de la tierra, trayendo consigo el aumento en áreas de tierras agropecuarias en un $59.2 \%$ equivalente a $480 \mathrm{ha}$.

\section{Uso actual del suelo en la microcuenca}

A continuación se presentan las características de los suelos de los dos sistemas principales en la microcuenca: Bl y Ta. (Ver mapa de confrontación del suelo. Mapa 1 uso actual).

\begin{tabular}{|l|l|l|l|l|}
\hline Clases de uso de suelo & Área_Ha & $\begin{array}{l}\text { \% con respecto al } \\
\text { superficie total de la } \\
\text { microcuenca }\end{array}$ & $\begin{array}{l}\text { Topografía } \\
\text { suelo }^{4}\end{array}$ \\
\hline Bosque latifoliado $(\mathrm{Bl})$ & 330 & 40.8 & 8201420 m.s.n.m & $25 \mathrm{~cm}$ \\
\hline Tierras agropecuarias (Ta) & 480 & 59.2 & $300-820$ m.s.n.m & $25 \mathrm{~cm}$ \\
\hline
\end{tabular}

Tabla2: Clases de uso de suelo, Fuente: Propia

3 No existen datos poblacionales que evidencien de cuanto fue el aumento, pero según las entrevistas realizadas a Florentín Ramírez y Francisco José afirman este aumento.

4 Entrevistas a los comunitaríos y visita en campo 
Según el uso actual del suelo, los con clase Bl son los menos representativos en la microcuenca, con un área de 330 ha y se ubican en zonas de mayores pendientes de 820 a 1420 m.s.n.m (ver mapa No2 Modelo altitudinal: Microcuenca Bilampí, pág. 30). En cambio, las superficies destinadas a actividades agrícolas y ganaderas ocupan un área de 480 ha, lo que equivale al 59\% de la superficie total del área en estudio, localizándose la misma sobre pendientes moderadas de 300 a 820 m.s.n.m, con tendencia a ascenso hacia la parte alta de la microcuenca.

La categoría Ta es una clase observada en mapas a nivel nacional, por ello en la microcuenca los históricos usos de suelo, la frágil situación socioeconómica de los comunitarios y las prácticas agropecuarias sin conservación de agua y suelo, han provocado el avance de la frontera agropecuaria generando impactos negativos en el cauce principal del río Bilampi.

\section{Confrontación del uso actual y uso potencial del suelo en el área de estudio}

La microcuenca presenta un índice de tierras sobre utilizada, correspondiente al 40\% (324ha) de la superficie explotada por encima del potencial que los suelos permiten, las cuales además son utilizadas en actividades agro productivas que no corresponden a su vocación. El uso subutilizado es de $21 \%$ (164). Estas áreas dedicadas a cultivos de cacao y musáceas es una alternativa de la pequeña central hidroeléctrica $(\mathbf{P C H})$ afectada a consecuencia de la sobreutilización a la que anteriormente estaban sometidos y que ha generado disminución del caudal hídrico.

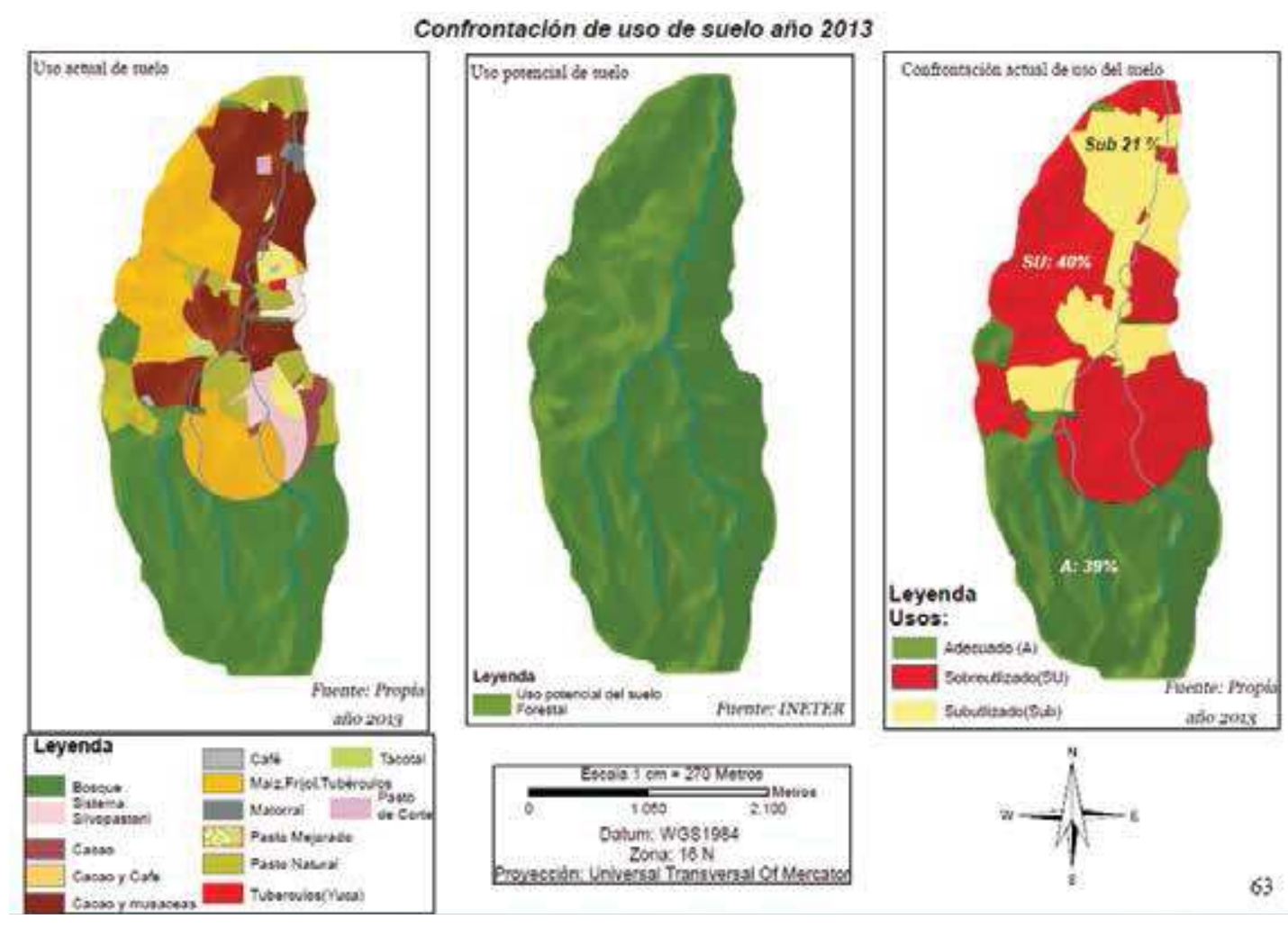

Mapa 5: Confrontación de uso de suelo año 2013 


\section{Impacto ambiental en el recurso hídrico de la microcuencia del río Bilampí}

\section{Implicaciones ambientales}

Uno de los impactos perceptibles en la microcuenca es la sobreutilización del suelo por encima de su uso potencial. De acuerdo al mapa elaborado por INETER se distingue que el ciento por ciento del suelo de la microcuenca es de uso forestal sin embargo, 450 ha de bosque latifoliado (Bl) han sido sustituidas por áreas de cultivos en un período de 30 años.

De acuerdo a los mapas elaborados en fechas anteriores, se observa la existencia de grandes hectáreas del bosque tropical latifoliado, hoy día por la influencia antrópica ha desaparecido, y con él, una gran diversidad de especies de plantas como: epifitas, helechos, criptógamas; árboles maderables como el cedro, pochote, guanacaste, ceiba y de animales como el Danto, Tigrillo, Saíno, Tucán de pico feliz, Pavas, Gavilán Blanco, Monos Congós y aves como el Quetzal, se encuentran en peligro de extinción.
A ello se le suma el impacto ambiental el cual se hace evidente con la disminución de la profundidad del agua y la contaminación del río Bilampí, generado por la desforestación, mal manejo de los suelos, implementación de sistemas productivos extensivos y la falta de alternativas para el uso de técnicas agrícolas adecuadas. Del área total de la microcuenca, el 59.2\% está desforestada, consecuencia de los sistemas productivos extensivos que se manifiesta principalmente en la parte baja y media, con tendencia a ascenso hacia la parte alta de la microcuenca

El mal manejo de las tierras en la microcuenca por parte de los productores, está vinculado al inadecuado uso potencial de los suelos. En la actualidad están siendo utilizados para cultivos de maíz, frijoles, tubérculos, musáceas y cacao, lo que conlleva al agotamiento, pérdida de fertilidad y sobreutilización de los suelos (Ver mapa $\mathrm{N}^{\circ}$ 5). A esta problemática los agricultores buscan como alternativa de solución el uso de técnicas tradicionales como la utilización de plaguicidas organoclorados y organofosforados los cuales contaminan las aguas del río Bilampí.

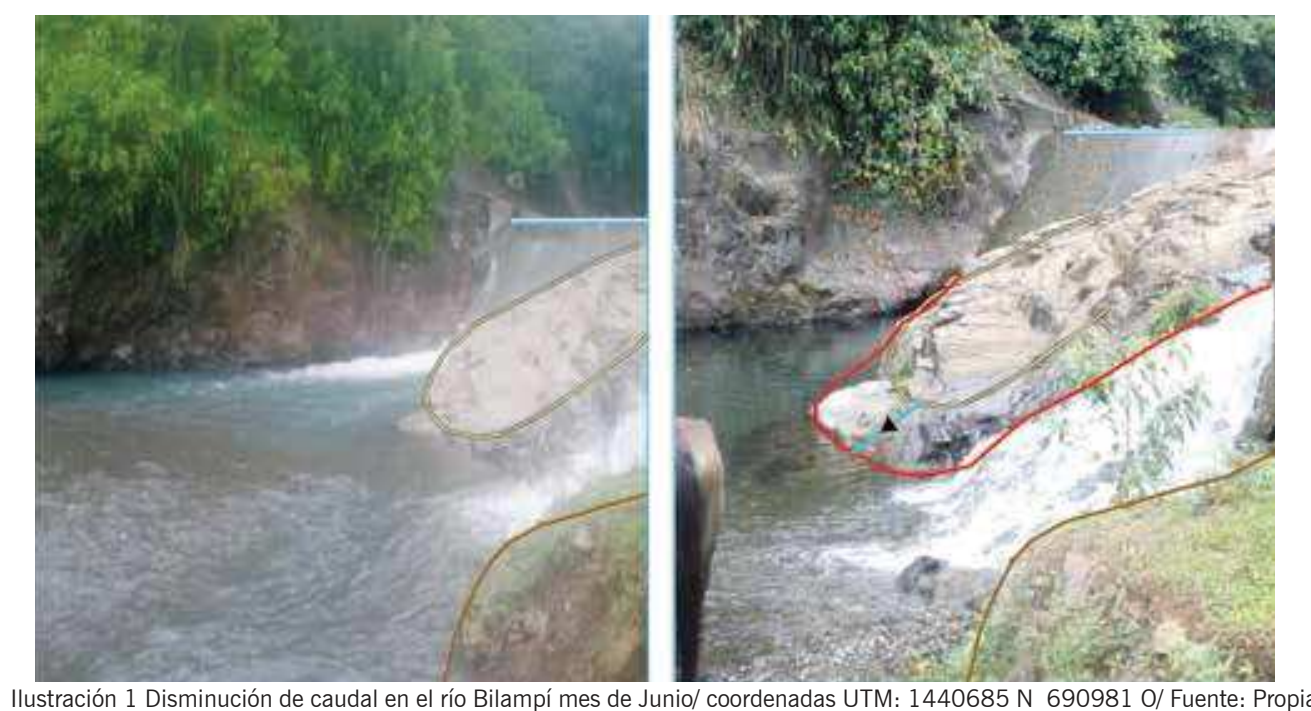


Mediante visitas in situ a la captación de agua para la $\mathrm{PCH}$, se obtuvieron las anteriores ilustraciones en la que se identifica los niveles de profundidad de las aguas del río Bilampí, la cual han variado en un período de dos años (2011-2013). La línea celeste marca el declive antes de la captación, observándose para el año 2011 abundante flujo de agua y dos años más tarde, la presencia de sedimentos naturales y la disminución de su caudal. Esto significa un notable cambio de aproximadamente $20 \mathrm{~cm}$ de agua, tomando en cuenta la altura del afloramiento rocoso marcado desde la línea color amarillo hasta la del color rojo. Otro rasgo que denota esta disminución es la línea café, donde se observa que la profundidad del espejo de agua en el año 2011 llegaba hasta ella, contrario a lo que sucede en la ilustración derecha del año 2013.
En la ilustración $\mathrm{N}^{\circ} 2$ se muestra el río Bilampí durante el recorrido en la parte baja de la microcuenca donde se comprueba el impacto que está sufriendo. En esta misma ilustración se observa la inexistencia de agua y la presencia de una vegetación tipo matorrales que no coincide con la vegetación de bosque latifoliado existente para el año 2001, lo cual es producto de la transformación sufrida en estos años a consecuencia del tipo manejo hecho por los productores y comunitarios en el área en estudio.

De acuerdo a las entrevistas, al no contar la comunidad con alcantarillado sanitario, ni de ningún sistema de manejo de las aguas servidas, el ciento por ciento de las familias dejan que las aguas residuales de las casas corran por la superficie del suelo, observándose la existencia de charcas en

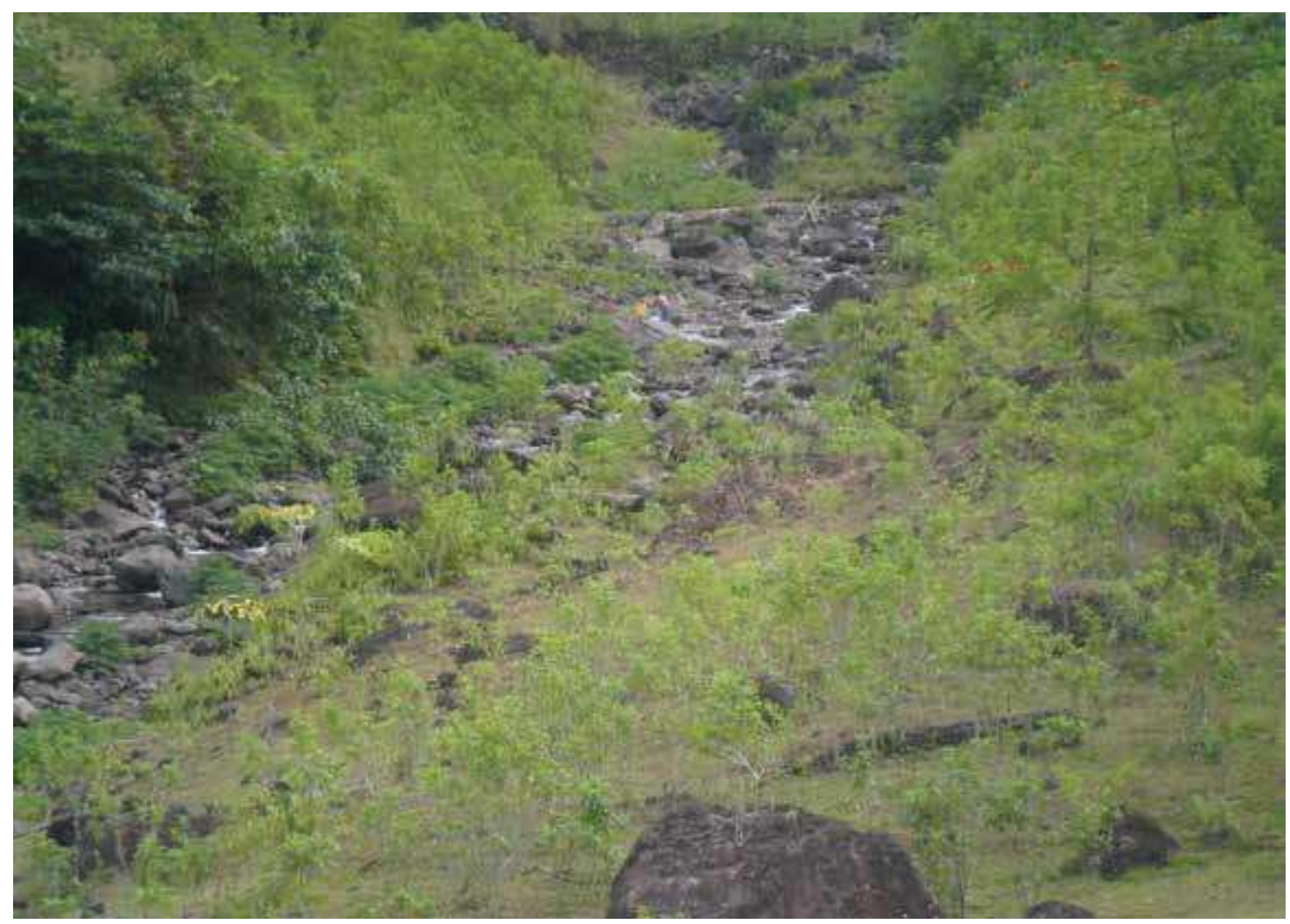

Ilustración 2. 
los patios, principalmente en el área de las cocinas. Otra de las implicaciones es la falta de recolección y tratamiento de la basura, lo cual es unos factores que más incidencia tiene en la contaminación del agua, por desechos de recipientes y empaques de plaguicidas los cuales son transportados y depositados por la escorrentía superficial en la ribera del río Bilampí. No se debe descartase la contaminación del ambiente por los animales domésticos no controlados en corrales, alimentándose algunos de ellos de excretas humanas.

La disminución del caudal del río Bilampí observada en las ilustraciones anteriores, ha hecho que la $\mathrm{PCH}$ y los agricultores ubicados en las riberas del río, siembren cacao como alternativa de solución para aminorar el impacto de las altas tasas de desforestación y evitar que el río siga perdiendo caudal y afecte de manera directa la producción de energía hidroeléctrica que abastece a nueve comunidades del municipio de Río Blanco.

Se observa que el áreas destinada a cultivos de cacao ubicado en la parte media-baja se encuentran distribuidos en dirección este y oeste a $20 \mathrm{~m}$ de la ribera del río, con extensiones de aproximadamente 150 ha equivalente al $18.5 \%$ de la superficie total de la microcuenca.

En visitas in situ se observó que los insumos de tipo agroquímico utilizado, es el gramoxone y plaguicidas organocloradoorganofosforado, todos ellos pertenecientes a la docena sucia (docena maldita) prohibido a nivel nacional e internacional. El primero se aplica cada cuatro semanas para mantener alejada a los insectos de la planta, expresaba Milton Ramírez productor de la microcuenca.

Es evidente que en gran parte de la microcuenca la influencia antrópica ha acelerado un proceso de meteorización, erosión y sedimentación de los suelos. De igual manera, el cambio de uso de suelo para las actividades productivas se ha dado en un marco de intensificación de uso de plaguicidas. El corto periodo de retorno de las aguas drenadas sumada a lo accidentada de la topografía de la microcuenca, facilita el transporte $y$ depósito de los residuos de plaguicidas en la parte bajas y muy probablemente se encuentren huellas de contaminación en la siguiente subcuenca o cuenca principal.

\section{Análisis del resultado en la muestra de agua de plaguicidas de organoclorados y organofosforados.}

Como un instrumento para identificar el grado de contaminación por plaguicidas en la microcuenca Bilampí, se tomaron muestras de agua, exactamente el 06 de junio del 2013 a las 8:40 am. En este ejercicio de campo se tuvo como factor adverso, las condiciones hidrometeorológicas, consecuencia de período de lluvia de Nicaragua bien establecido para esta época. Aún en estas condiciones, algún indicio de pluma contaminante provocada por los usos de plaguicidas tipo organoclorados (OC) y organofosforados (OF) se encuentran en el río. La muestra por recomendaciones técnicas fueron tomadas en la parte baja del río, bajo la técnica de conveniencia, de acuerdo a los siguientes criterios: influencia directa de los ciudadanos sobre el recurso y usos del agua (consumo humano, actividades domésticas y agropecuarias).

El período lluvioso conlleva, al aumento del caudal del río influyendo en la circulación del agua de forma natural y rápida. Esta situación hace que los residuos de plaguicidas OC y OF, sean transportados más abajo de la toma de muestra de agua, 
lo que implica la posible presencia en la microcuenca del rio el Diamante.

Una muestra de agua no es confiable en su totalidad para determinar la presencia y cantidad de plaguicidas OC y OF, debió de tomarse en cuenta otros elementos de estudio como la observación in situ, las características socioeconómica, el avance de la frontera agropecuaria, los cambios de uso de suelo, la utilización de plaguicidas en cultivos en la microcuenca. De igual manera, el avance tecnológico permitió la utilización del modelo hidrológico donde se muestra la dirección y desplazamiento de las trazas de contaminación hacia el río Bilampí.

A ello debe de sumarse las entrevistas realizadas a los agricultores y al promotor ambiental Gustavo Soza González, quien confirma la persistencia de prácticas de lavado de bombas usadas para la fumigación de los cultivos, a la orilla del rio Bilampí. Esta costumbre es habitual entre los productores, realizándose al inicio y después de la jornada laboral. También existe la cultura de la preparación de estas sustancias a las orillas de estas fuentes. De acuerdo a lo anterior, no se descarta la posible presencia de plaguicidas organoclorados (OC) y organofosforados (OF). Bajo recomendaciones del CIRAUNAN, la muestra se tomó a 0.5 metros de fondo y de la superficie, evitando partículas de sedimentos en suspensión Antes de la toma de la muestra, se marcó el frasco con rotulador resistente al agua, el nombre de la muestra y en una ficha técnica se anotaron los siguientes datos:

\begin{tabular}{|l|l|}
\hline \multicolumn{1}{|c|}{$\begin{array}{c}\text { Nombre de la } \\
\text { muestra }\end{array}$} & \multicolumn{1}{c|}{ Río Bilampí 1 } \\
\hline Tipo & $\begin{array}{l}\text { Plaguicidas } \\
\text { Organoclorados y } \\
\text { organofosforados }\end{array}$ \\
\hline $\begin{array}{l}\text { Municipio, } \\
\text { departamento }\end{array}$ & Río Blanco, Matagalpa \\
\hline Coordenadas & 144147 N/691481 O \\
\hline Altura m.s.n.m & $\begin{array}{l}340 \text { (parte baja de la } \\
\text { microcuenca) }\end{array}$ \\
\hline Fecha de muestreo & 06 de junio 2013 \\
\hline Hora & $8: 40$ am \\
\hline
\end{tabular}

Tabla3: Datos de la toma de muestra de agua, Fuente Propia

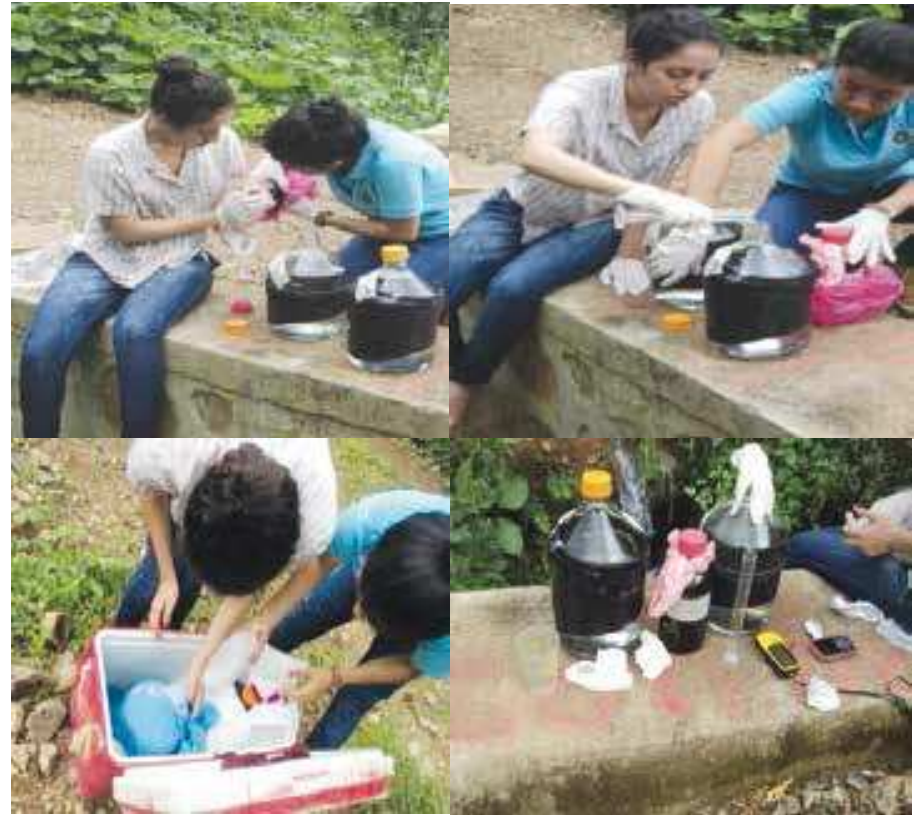

Ilustración 2 Procedimientos en la toma de muestra de agua
A tres litros de agua se le agregó $50 \mathrm{ml}$ de una mezcla de la sustancia química hexano (foto 1 y 2 ) y se colocó en un termo con hielo para preservar la muestra durante su transportación, siendo analizadas por el CIRA UNAN antes delas 24 horas. La muestra de agua fue recogida en la parte baja de la microcuenca del río Bilampí, bajo la técnica de conveniencia, de acuerdo a los siguientes criterios: influencia directa de los ciudadanos sobre el recurso y usos del agua (consumo humano, actividades domésticas y agropecuarias). 


\section{Conclusiones}

Al finalizar la investigación y evaluar la dinámica socioeconómica, el uso de suelo multitemporal-espacial y su impacto ambiental en el recurso hídrico de la microcuenca del río Bilampí, se concluye con lo siguiente:

1. Al ser la microcuenca Bilampí de forma oval redonda a oval oblonga $\mathrm{y}$ con pendientes mayores a 500 m.s.n.m, aumenta las probabilidades de crecidas súbitas e inundaciones en la microcuenca. Por tanto el arrastre de sedimentos orgánicos e inorgánicos procedentes de la erosión de las tierras de cultivo con restos de plaguicidas, se depositan en la parte media y baja de la microcuenca provocando la contaminación de sus aguas.

2. El53\% de la población económicamente activa en la microcuenca se dedica a actividades agropecuarias, empleando técnicas tradicionales como el uso principalmente de plaguicidas de tipo organoclorado y organofosforado, agricultura extensiva sin conservación de agua y suelo. La exposición constante ante este tipo de plaguicidas está afectando la salud de los comunitarios generando enfermedades dérmicas y respiratorias y no se descarta la aparición de enfermedades crónicas en un futuro como: cánceres, leucemia, necrosis de hígado, malformaciones congénitas, neuropatías periféricas, a veces solo malestar general, cefaleas persistentes.

3. La tasa de cambio de uso de suelo en la microcuenca ha variado paulatinamente desde el año de 1983 al 2013, pasando de 780ha de bosque latifoliado a 330ha esto significa, una disminución de 450ha que fueron sustituidas por áreas para cultivos de maíz, frijoles, cacao tubérculos, musáceas y pastos, generando un avance de la frontera agropecuaria hacia la parte alta de la microcuenca e impactando en la contaminación y disminución del caudal del río Bilampí. La implementación de sistemas extensivos de producción agrícola y pecuaria, fomentan y contribuyen a la reducción de las áreas de bosque.

4. Se elaboró el mapa de uso de suelo del año 2013 en la microcuenca, en el cual se observan 14 clases de uso ubicados arriba de la cota de elevación de los 500 m.s.n.m, infringiendo lo establecido en el plan del comanejo para el área protegida del cerro Musún. En la parte alta de la microcuenca predomina la categoría Bosque latifoliado y algunos parches de parcelas cultivadas con maíz, frijoles y tubérculos, sin embargo esta últimas prevalecen en la parte media y baja.

5. La microcuenca presenta un índice de tierras sobreutilizada, correspondiente al $40 \%$, el uso adecuado es39\% y el subutilizado es de $21 \%$, el primer dato indica que está siendo explotada por encima del potencial establecido (forestal). La subutilización corresponde a cultivos de cacao y musáceas, donde los productores y la $\mathrm{PCH}$ se vieron obligados a plantarlas como medida ante la anterior sobreutilización en las que se encontraban estas tierras.

6. El cacao siendo una de las alternativas en la conservación de suelo y el agua en su mayoría se encuentran distribuidas en la parte media y baja de la microcuenca, sin embargo está incidiendo en la contaminación de las aguas debido a que se están fumigando con gramoxone cada cuatro semanas, 
sin tomar en cuenta que está prohibido a nivel nacional e internacional.

7. Durante el análisis de la muestra de agua que se recopiló en el período lluvioso (mes de junio), no se encontraron hallazgos de plaguicidas de tipo organoclorados y organofosforados, sin embargo, no se descarta la presencia de este tipo de agroquímicos debido a que in situ se observa el uso intensivo de plaguicidas, malas prácticas agrícolas, cambio de vocación de suelos y la dirección de trazas de contaminación que de acuerdo al modelo hidrológico todo llegan a depositarse en el recurso hídrico de la microcuenca.

\section{Referencias Bibliográficas}

Cruz Méndez Oscar, Molina Marcia Juniette, (1998), Evaluación de la calidad hidroquimica del Río San Juan y sus Ríos tributaríos, CIRA-UNAN, (s.n)

ENACAL (Empresa de acueductos y alcantarillados), Microcuenca hidrográficas identificadas para el suministro de agua potable.

ENACAL, (2006), sobre el recurso agua y su situación en Nicaragua, abc segunda edición..

Hernández Silvia, (2007), Diagnostico de la calidad de agua que drenan al lago Cocibolca en el período de 1994-97,20023. CIRA-UNAN. (s.n)

MARENA (Ministerío del Ambiente y los Recursos Naturales), Managua, 2010 caracterización de la cuenca no. 55 río grande de Matagalpa.
Méndez Ramírez Ignacio, Guerrero Nahimira Delia, Altamirano Moreno Laura y Sosa de Méndez Cristina (2008), El protocolo de investigación lineamientos para su elaboración. (s.n)

Montiel Rodríguez Sonia et.al, (1982), Geografía económica General. (s.n)

Sampieri Hernández Roberto, Collado Fernández Carlos, Lucio Baptista Pilar (2006), Metodología de la investigación, cuarta edición, McGraw-Hill interamericana.

\section{Artículos de Revistas científicas}

Salas A.V, Pinedo A. C, Viramontes O.O, Gonzalez V.A, Quintana M. R,(2010) Morphometric of the Nazas-Rodeo riverWatershed in Durango, Mexico, ByGeographicaltechnologysystems. Revista Electrónica de medio ambiente y desarrollo sustentable. Pag 23. Recuperado el 28 de octubre del 2012.http://tecnociencia.uach.mx/ numeros/v5n1/data/Morfometria de_la_cuenca_del_ríoNazas_Rodeo_ aplicando_tecnologia_geoespacial_ en_Durango_Mexico.pdf.

Viramontes O.O, Luis Fernando Escoboza. G L. F, Pinedo-Á.C, Pinedo-Á.A ,Reyes. V.M, Román.CJ.A,Pez A.M(2008) Morphometric of San Pedro Basin, Conchos, Chihuahua. Revista Electrónica de medio ambiente y desarrollo sustentable Pág. 23. Recuperado el 28 de octubre del 2012. http://tecnociencia.uach. $\mathrm{mx} / \mathrm{numeros} / \mathrm{v} 1 \mathrm{n} 3 / \mathrm{data} / 5$ morfometria-de-la-cuenca.pdf.

1 Entrevistas a los comunitaríos y visita en campo 